EPJ Web of Conferences 49, 15005 (2013)

DOI: $10.1051 /$ epjconf/20134915005

(C) Owned by the authors, published by EDP Sciences, 2013

\title{
Searches for a W', contact interactions and lepton resonances with CMS
}

\author{
Arnaud Gay ${ }^{1, a}$ on behalf of the CMS collaboration \\ ${ }^{1}$ IIHE-ULB Boulevard du Triomphe, 21050 Bruxelles Belgique
}

\begin{abstract}
We present the searches for W', contact interactions and lepton resonances at CMS. No evidence has been found so far and limits are set on various models.
\end{abstract}

\section{Introduction}

New heavy gauge bosons such as the W' and Z' are predicted in many models of new physics beyond the standard model (SM). In the sequential standard model (SSM) [1] an additional $\mathrm{U}(1)$ group is included and the W' and Z' boson are considered to be heavy analogues of the $\mathrm{W}$ and $\mathrm{Z}$, respectively. They are assumed to be narrow resonances with decay modes and branching fractions similar to those of their SM counterparts. Other models such as superstring inspired $E_{6}$ models have more complex group structures, $E_{6} \rightarrow S O(10) \times U(1)_{\psi}$, with corresponding neutral gauge bosons denoted as $Z_{\psi}^{\prime}$. Heavy resonances also arise in models with extra-dimensions, such as Kaluza-Klein graviton excitations in the Randall-Sundrum (RS) model. This model has two free parameters: the mass of the first graviton excitation and the coupling $k / \bar{M}_{P l}$, where $\mathrm{k}$ is the curvature of the extra dimension and $\bar{M}_{P l}$ is the reduced effective Planck scale.

The variety of observed quark and lepton flavors and their mass hierarchies suggest a substructure. In order to confine the constituents (often referred to as "preons") and to account for the properties of quarks and leptons, a new strong gauge interaction, metacolor, is introduced. Below a given interaction scale $\Lambda$ the effect of the metacolor interaction is to bind the preons into metacolor-singlet states. For parton interaction $\hat{s}$ values that are much less than the $\Lambda$ scale the metacolor force will manifest itself in the form of a flavor-diagonal contact interaction [2, 3] (CI).

\section{Search for leptonic decays of W' bosons}

The main observable in this search [4] is the transverse mass $M_{\mathrm{T}}$ of the lepton- $E_{T}^{\text {miss }}$ system. The $E_{T}^{\text {miss }}$ comes from the undetected neutrino. In W' decays, the lepton and $E_{T}^{m i s s}$ are expected to be almost back-to-back in the transverse plane, and balanced in transverse energy. Candidate events are therefore selected through a requirement on the ratio of the lepton $p_{\mathrm{T}}$ and the $E_{T}^{\text {miss }}, 0.4<p_{\mathrm{T}} / E_{T}^{\text {miss }}<1.5$. A requirement is also imposed on the angular difference

\footnotetext{
a e-mail: arnaud.gay@cern.ch
}
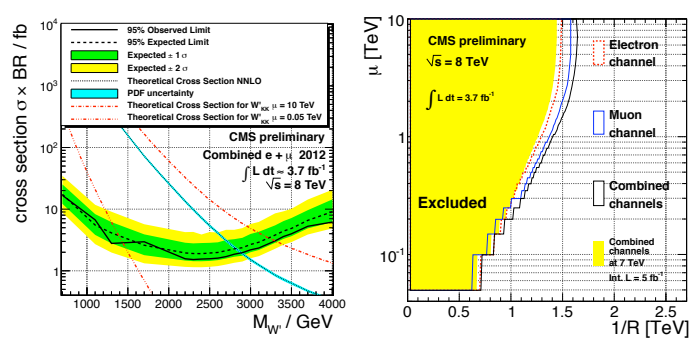

Figure 1. Left: Excluded W'cross section for the combination of 2012 electron and muon channel. Right: 95\% CL limits on the split-UED parameters $\mu$ and $1 / R$.

in the transverse plane of the lepton and $E_{T}^{m i s s}$ direction, $\Delta \phi_{\ell, v}>0.8 \times \pi$. The primary source of background (BG) for all these signals is the off-peak, high transverse mass tail of the standard model (SM) $W \rightarrow \ell v$ decays. Other important BG arise from QCD multijet, $t+\bar{t}$, and Drell-Yan events. Di-bosons (WW, WZ, ZZ) decaying to electrons, muons, or taus were also considered. Multijet BG is largely suppressed by the event selection requirements. The $M_{T}$ distribution is fitted with the function $f\left(M_{\mathrm{T}}\right)=\frac{a}{\left(M_{\mathrm{T}}+b\right)^{c}}$ to estimate the BG. A $W^{\prime} \rightarrow e v$ or $W^{\prime} \rightarrow \mu v$ signal is expected to manifest itself as an excess over the SM expectation in the tail of the $M_{\mathrm{T}}$ distribution. No significant excess has been observed in the 3.7/fb of data at $8 \mathrm{TeV}$ analysed. The observed highest transverse mass events have $M_{\mathrm{T}}=2.3(1.3) \mathrm{TeV}$ in the electron (muon) channel. Upper limits on the production cross section times the branching fraction $\sigma_{W^{\prime}} \times \mathcal{B}\left(W^{\prime} \rightarrow l v\right)$, with $l=e$ or $\mu$ are thus set, using a Bayesian method [5] with a uniform prior probability distribution for the signal cross section. The number of data events above an optimized for the best expected exclusion limit transverse mass threshold $M_{\mathrm{T}}^{\mathrm{min}}$ was compared to the expected number of signal and BG events. Systematic uncertainties on the signal and BG yield were included via nuisance parameters with a log-normal prior distribution. Using the central value of the theoretical cross section times the branching fraction, we exclude at $95 \%$ confidence level (CL) the exis- 
tence of a W'with SM-like couplings of masses less than $2.75 \mathrm{TeV}$ (compared with an expected limit of $2.65 \mathrm{TeV}$ ) in the muon and $2.60 \mathrm{TeV}$ (compared to $2.70 \mathrm{TeV}$ expected) in the electron channel. In the latter the observed limit is below the expected due to the event at $M_{\mathrm{T}}=2.3$ $\mathrm{TeV}$. The results of the individual channels are combined assuming independent uncertainties (no correlation) apart from the luminosity uncertainty which is fully correlated. The combined limit is $2.85 \mathrm{TeV}$. With $5 \mathrm{fb}^{-1}$ of data at $\sqrt{s}=7 \mathrm{TeV}$ this limit was $2.5 \mathrm{TeV}$. The combination of 2011 and 2012 datasets was performed but didn't improve the sensitivity. The observed limits can be reinterpreted in terms of the second Kaluza-Klein excitation $W_{\mathrm{KK}}^{2}$ mass in a specific framework of universal extra dimensions with bulk mass fermions. The $W_{\mathrm{KK}}^{2}$ is the lowest state that can couple to SM fermions and has the same final state as the SM-like W'. Since it has even parity it can be produced singly. $W_{\mathrm{KK}}^{2}$ has been excluded for masses below 1.4 (3.3) $\mathrm{TeV}$, assuming a bulk mass parameter $\mu$ of 0.05 (10) $\mathrm{TeV}$. These lower limits on the mass can be directly translated to bounds on the split-UED parameter space $[1 / R, \mu]$ as shown in Figure 1.

\section{Search for exotic resonances in VZ}

The study of the di-vector boson $V V$ production $(V=$ $W, Z$ ) plays a special role in testing theories proposed as an alternative to the Higgs mechanism. Several models predict the existence of new particles that couple to two vector bosons. A few examples are Randall-Sundrum (RS) gravitons coupling to $Z Z$ and $W W$, or technimesons coupling to $W Z$. Limits from previous searches and from indirect bounds place the masses of these proposed RS resonances at or above the $\mathrm{TeV}$ scale. We present a search [6] for heavy resonances decaying to a $Z \rightarrow \ell \ell(\ell=\mu, e)$ and a vector boson $(V)$ decaying hadronically. We consider the Sequential Standard Model (SSM) $W^{\prime} \rightarrow W Z \rightarrow \ell \ell j j$ and $G_{R S} \rightarrow Z Z \rightarrow \ell \ell j j$ decay chains as benchmark models, without enforcing the specific nature of the produced particle in the analysis. This allows us to interpret our results in different theoretical frameworks, as for instance the RS model. $Z$ candidates are formed from two same-flavor, opposite-sign leptons and selected by requiring their invariant mass to be in the $70<m_{Z}<110 \mathrm{GeV}$ mass range and with a transverse momentum $p_{T}^{Z}>150 \mathrm{GeV}$. We reconstruct the $V \rightarrow q q$ candidate as a single jet (as the resonance is postulated to be heavy, the quarks are boosted and close to each other), well separated from the two leptons forming the $Z$ candidate: $\Delta R($ jet,$\ell)>1.0$. We request the jet mass, $m_{j}$, computed from the vectorial sum of the four-momenta of the constituent particles to be in the $65<m_{j}<120 \mathrm{GeV}$ mass range. We also request the $V$ candidate transverse momentum, $p_{T}^{V}$, to be larger than $250 \mathrm{GeV}$. Once the $Z \rightarrow \ell \ell$ and (mono-jet) $V \rightarrow q q$ candidates have been reconstructed, we combine their four momenta to compute the mass of the mother particle, $m_{V Z}$. This variable is used to evaluate the hypothesis of the signal presence in the analyzed datasets. According to simulation, the dominant $(\sim 90 \%)$ BG in this analysis after

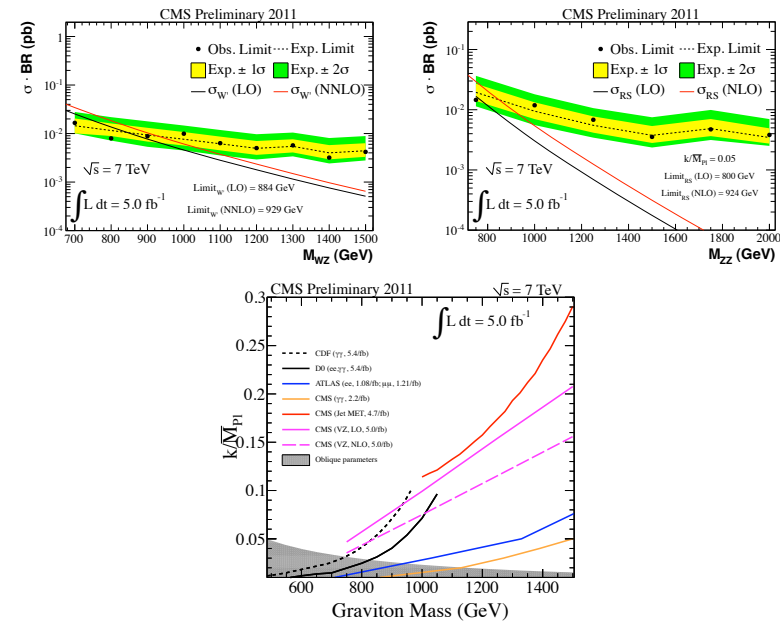

Figure 2. Exclusion limits for a counting experiment and comparison with the theoretical predictions in W'(top left) and RS Graviton (top right) models for the combination of electron and muon channels. Bottom: Interpretation of the limits in the $m_{R S}{ }^{-}$ $k / \bar{M}_{P l}$ plane.

all selection cuts is the inclusive $Z$ production (" $Z+$ jets"), with additional contributions from $t \bar{t}+$ jets and the continuum SM two-vector boson production ( $W Z$ and $Z Z$ ). The shape and the overall normalization of the expected BG $m_{V Z}$ distributions are derived from data, with additional cross-checks carried out with the inclusive simulated BG samples. No significant excess of events is observed, with the largest deviation appearing in the $\sim 900 \mathrm{GeV}$ region in the muon channel. The systematic uncertainties considered in this analysis can be divided into two main categories: the uncertainty in the determination of the SM BG and the uncertainty in the expected yields of signal events. For the expected signal yields, the systematic uncertainties that we considered are on the luminosity measurement, the JES, the parton density function (PDF) and trigger and reconstruction efficiencies. Finally, we assign a $9 \%$ systematic uncertainty on the $V$ mass cut efficiency. We employ the modified frequentist CLs statistical method and a series of search windows corresponding to different mass hypotheses to search for exotic $V Z$ resonances. Each mass window is optimized to give the best exclusion limit. We calculate $95 \%$ C.L. exclusion limits (Fig. 2) on the combined $\sigma(V Z) \times \mathcal{B}(V \rightarrow q q, Z \rightarrow \ell \ell, \ell=e, \mu)$ product for the two final states under study (separately and combined) as a function of the mass of the hypothetical resonance. The limit setting is performed by looking for an excess over the expected BG in the $V Z$ mass distributions for the two channels separately. We exclude W'bosons with masses between 700 and $929 \mathrm{GeV}$ in the SSM at 95\% C.L.The exclusion limit calculated in the RS Graviton model is for masses $\left(m_{R S}\right)$ between 700 and $924 \mathrm{GeV}$ for $k / \bar{M}_{P l}=0.05$. Assuming the resonance width is much smaller than the experimental resolution for the range of $k / \bar{M}_{P l}$ considered here, the limit can be extended in the $m_{R S}-k / \bar{M}_{P l}$ plane, by rescaling the cross section as a function of $k / \bar{M}_{P l}$ and assuming that the signal efficiency remains the same. The 
result is shown in Fig. 2. These results are not as competitive as the ones derived in the $\gamma \gamma$ and $\ell \ell$ channel searches $\left(m_{R S}>1500 \mathrm{GeV}\right)$. However, one should consider that those are derived assuming a democratic RS model. In more recent studies, RS models with SM fields propagating have been proposed, in which case the RS graviton does not couple to fermions. This opens the possibility to an enhancement of the branching ratios for final states with $V$ pairs, as well as the interest to study large values of $k / \bar{M}_{P l}[7]$.

\section{Search for resonances decaying to ee $/ \mu \mu$}

For both the dimuon and dielectron final states, two isolated and same-flavor high-pt leptons were required [8]. The most prominent SM BG process (either directly or via $\tau \tau)$ is Drell-Yan production $\left(Z / \gamma^{*}\right)$; there are also contributions from $\bar{t} \bar{t}, \mathrm{tW}$, and diboson processes. In addition, jets may be misidentified as leptons and contribute to the BG through multijet and vector boson plus jets final states. In the final dilepton spectra, the BG component from SM processes is found by fitting an appropriate function to the data. Studies of the BG components were performed in order to verify that its assumed composition is correct but are not used directly to estimate the magnitude of the BG. In absence of signal, limits are set on the possible contributions from narrow heavy resonances. A key feature of the statistical procedure is that it requires no knowledge of the integrated luminosity as the BG estimations are normalized to the data in regions where SM processes would dominate any potential signal and the limits are set on the ratio of the products of cross sections and branching fractions: $R_{\sigma}=\frac{\sigma\left(p p \rightarrow Z^{\prime}+X \rightarrow \ell \ell+X\right)}{\sigma(p p \rightarrow Z+X \rightarrow \ell \ell+X)}$. This eliminates the uncertainty in the integrated luminosity and reduces the dependence on experimental acceptance, trigger, and offline efficiencies. The ratio of acceptances of the new boson to the $Z$ is calculated using Monte Carlo simulation with the assumption that the new physics comes from a resonance of spin 1. The extended unbinned likelihood function for the spectrum of dilepton invariant mass values $m$ above $200 \mathrm{GeV}$ is based on a sum of analytic probability density functions (pdfs) for the signal and BG shapes. The pdf $f_{\mathrm{S}}(m \mid \Gamma, M, w)$ for the resonance signal is a Breit-Wigner of width $\Gamma$ and mass $M$ convoluted with a Gaussian resolution function of width $w$. The width $\Gamma$ is taken to be that of the $Z_{\psi}^{\prime}$. This width is sufficiently small that the detector resolution dominates. A BG pdf $f_{\mathrm{B}}$ was chosen and its shape parameters fixed by fitting to the simulated Drell-Yan spectrum in the mass range $200<m_{\ell \ell}<2500$ $\mathrm{GeV}$. No significant difference in shape is observed when fitting just the Drell-Yan and the Drell-Yan and all other SM BGs. The functional form used for the BG is $m^{-\kappa-\alpha m}$. In absence of signal, upper limits are calculated using the Bayesian technique. The dominant uncertainty in the analysis is that on $R_{\epsilon}$, the ratio of selection efficiency times detector acceptance for $Z^{\prime}$ decay to that for $Z$ decay. This uncertainty is $8 \%$ for the dielectron channel and $3 \%$ for the dimuon channel. These values reflect our current understanding of the detector acceptance (including the impact
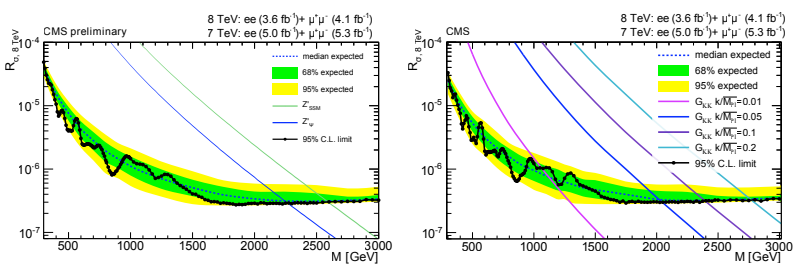

Figure 3. Upper limits as a function of resonance mass $M$ on the production ratio $R_{\sigma}$ of cross section times branching fraction into lepton pairs for $Z_{S S M}^{\prime}, Z_{\psi}^{\prime}$ boson production (left) and $G_{K K}$ (right) to the same quantity for $Z$ bosons for the combined dilepton $7+8$ $\mathrm{TeV}$ data.

of PDF uncertainties on the acceptance) and the reconstruction efficiency turn-on at low mass, as well as their values at high mass. The largest contribution to the uncertainty on $R_{\epsilon}$ comes from the uncertainty in the lepton reconstruction efficiency at high mass. To further improve the exclusion limits, the dataset taken until june 2012 at $\sqrt{s}=8 \mathrm{TeV}$ is combined with the one collected in 2011 at $\sqrt{s}=7 \mathrm{TeV}$. To account for the different center-of-mass energies, the predicted ratio of $R_{\sigma}$ for a quark-antiquark annihilation at $\sqrt{s}=7 \mathrm{TeV}$ and at $8 \mathrm{TeV}$ is used to convert the published $7 \mathrm{TeV}$ limits to $8 \mathrm{TeV}$. The additional uncertainty this introduces on $R_{\sigma}$ is negligible compared to existing uncertainties affecting this variable. Figure 3 shows the observed and expected upper limits on the production ratio $R_{\sigma}$ of the cross section times branching fraction of a Z' boson and $G_{K K}$ relative to that for a $\mathrm{Z}$ boson for the dimuon and dielectron combination for the 7 and $8 \mathrm{TeV}$ datasets combined. The figures also show the predicted cross section times branching fraction ratios for $Z_{S S M}^{\prime}, Z_{\psi}^{\prime}$ and $G_{K K}$ production. A $Z_{S S M}^{\prime}\left(Z_{\psi}^{\prime}\right)$ with a mass less that 2590 (2260) $\mathrm{GeV}$ is thus excluded. The $G_{K K}$ are excluded below 2390 (2030) GeV for $\mu=0.1(0.05) \mathrm{TeV}$.

\section{Search for resonances decaying to $\tau^{+} \tau^{-}$}

Taus can decay into lighter leptons plus neutrinos (referred to as $\tau_{\ell}, \ell=e, \mu$ ) and into a hadronic system (referred to as $\tau_{h}$ ). Four ditau final states have been studied [9]: $\tau_{e} \tau_{\mu}, \tau_{e} \tau_{\mathrm{h}}, \tau_{\mu} \tau_{\mathrm{h}}$, and $\tau_{\mathrm{h}} \tau_{\mathrm{h}}$. The signature is two high $p_{T}$, oppositely-charged, isolated, and almost back-to-back tau candidates. Ditau pairs are formed from oppositelycharged candidates separated in $\Delta R$ space $(\Delta R>0.7)$. In addition, the back-to-back requirement on the ditau pairs is imposed by selecting candidates with $\cos \Delta \phi\left(\tau_{1}, \tau_{2}\right)<$ -0.95 , where $\Delta \phi\left(\tau_{1}, \tau_{2}\right)$ is the difference in the azimuthal angle between the $\tau$ candidates. The presence of multiple neutrinos in the final state, which escape CMS undetected, preclude us from fully reconstructing the mass of the ditau system. To more effectively distinguish between lower mass BGs from tau lepton pairs from new massive resonant particle production, the visible tau decay products and the $E_{T}^{\text {miss }}$ are used to reconstruct the partial mass. To estimate the BG contributions in the signal region, data-driven techniques are employed wherever possible. In cases where a complete data-driven approach is 


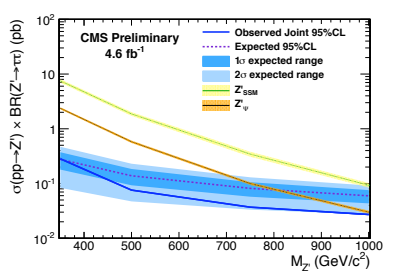

Figure 4. Combined 95\% CL upper limits on the cross section as a function of $Z^{\prime}$ mass.

not possible, data-to-MC scale factors are used to correct the expected contributions obtained from the simulated samples. The largest BG sources are from $\mathrm{W}+\mathrm{jet}(\mathrm{s})$ and Drell-Yan production for $\tau_{e} \tau_{\mu}$ and $\tau_{\ell} \tau_{\mathrm{h}}$ final state, and QCD for $\tau_{\mathrm{h}} \tau_{\mathrm{h}}$. However, the contamination from these BGs is small in the mass region essential for this analysis. The unbiased BG shapes can not be evaluated with the data available. The BG shapes are taken from Monte Carlo simulation validated in the control regions and normalized to the values obtained from the data-driven estimates. The main source of systematic uncertainty results from the estimation of the BG contributions that are dominated by the statistical uncertainty of the data used in the control regions. The contamination from other BGs in these control regions have a negligible effect on the systematic uncertainty. These uncertainties are in the range of 6 to 14\%. Systematic effects associated with hadronic $\tau$ identification are extracted from a global fit of the visible ditau mass and constraining the production cross section to the measured cross section in $\mathrm{Z} \rightarrow e^{+} e^{-}$and $\mathrm{Z} \rightarrow \mu^{+} \mu^{-}$ decay channels. This uncertainty amounts to $6.8 \%$ per tau leg. Uncertainties that contribute to the ditau mass shape variations include tau (2\%) and lepton (1\%) energy scale, and uncertainties on the $E_{T}^{m i s s}$ scale that is used for the $M\left(\tau_{1}, \tau_{2}, E_{T}^{\text {miss }}\right)$ mass reconstruction. Additional uncertainties that contribute to the mass shape are due to small statistics of the simulated samples in the high mass regions that lead to systematic uncertainties in the fit parameters. The scale uncertainties contribute via the jet energy scale (2-5\% depending on $\eta$ and $p_{T}$ ) and unclustered energy scale (10\%), where unclustered energy is defined as the energy found "outside" the reconstructed leptons and jets with $p_{T}>10 \mathrm{GeV} / \mathrm{c}$. The observed mass spectra do not reveal any evidence for $\mathrm{Z}^{\prime} \rightarrow \tau^{+} \tau^{-}$production. Using the CLs methodology an upper limit on the cross section $\sigma\left(\mathrm{pp} \rightarrow Z^{\prime}\right) \times \mathrm{BR}\left(Z^{\prime} \rightarrow \tau^{+} \tau^{-}\right)$at $95 \% \mathrm{CL}$ as a function of the mass for each ditau final state is calculated taking into account all systematic uncertainties. The final combined limit is obtained using a joint likelihood, where the systematic uncertainties, incorporated as nuisance parameters, have been marginalized. Combining all four final states (Figure 4) we can exclude $Z_{S S M}^{\prime}$ and $Z_{\psi}^{\prime}$ with SM couplings with masses less than $1 \mathrm{TeV} / \mathrm{c}^{2}$.

\section{Searches for contact interactions}

CI can be searched in $\mu^{+} \mu^{-}$final states [10]. Interferences (either constructive or destructive) with SM Drell-
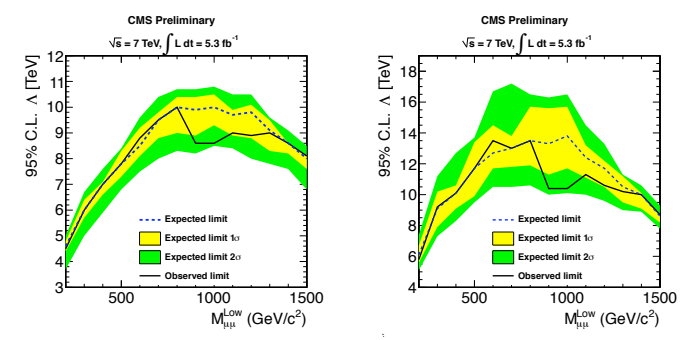

Figure 5. Observed and expected limits as a function of $M_{\mu \mu}^{L o w}$ for destructive (left) and constructive (right) interference.

Yan (DY) dimuon production must be taken into account and the processes contributing to the cross section are denoted collectively by "CI/DY". This analysis is restricted to the left-left isoscalar model LLIM where all of the initial state quarks are assumed to be composite. This model is the conventional benchmark for CI in the dilepton channel. The analysis of CI events is limited to a dimuon mass range from 200 to $2000 \mathrm{GeV} / \mathrm{c}^{2}$. The lower limit is enough above the Z-peak so that a deviation from DY production is observable, while the upper limit is chosen large enough to include all events that could be produced for values of $\Lambda$ accessible with this dataset and not excluded by previous measurements. The minimum mass $M_{\mu \mu}^{\text {Low }}$ required in the analysis is varied between the lower and upper limits to optimize the limit on $\Lambda$. The non-zero BGs, in decreasing order of importance, are $t \bar{t}$, diboson $(W W / W Z / Z Z), W$ (including $W+$ jets, $\bar{t} W$, and $t W$ ), and $Z \rightarrow \tau \tau$. The QCD BG is negligible. Event yields corresponding to the integrated luminosity of the data are predicted as a function of $\Lambda$ and $M_{\mu \mu}^{L o w}$, including the contributions from the CI/DY process and non-DY SM BGs. The expected and observed $95 \%$ CL lower limits on $\Lambda$ are determined using the $C L_{s}$ modified frequentist procedure, taking the profile likelihood ratio as a test statistic. The expected mean for the number of signal events is the total number of events expected using a given $\Lambda$ less the total number of events expected using the DY process. The expected mean for the number of $\mathrm{BG}$ events is the total number of events using the DY process. The systematic uncertainties on integrated luminosity, acceptance, and expected BG are included as nuisance parameters in the limit setting procedure. The acceptance uncertainty is dominated by the difference between acceptances determined using DY and CI/DY simulations. The uncertainty in BG is dominated by the effect of PDF variations. The observed and expected lower limits on $\Lambda$ at 95\% CL as a function of $M_{\mu \mu}^{L o w}$ for destructive and constructive interference are shown in Figs. 5. For both cases, the sensitivity to $\Lambda$ is maximal for $M_{\mu \mu}^{L o w}$ in the middle of the range studied. In both cases we select minimum mass of $700 \mathrm{GeV} / \mathrm{c}^{2}$, resulting in an observed (expected) limit of $9.5 \mathrm{TeV}(9.8 \mathrm{TeV})$ for destructive interference and 13.0 $\mathrm{TeV}(13.0 \mathrm{TeV})$ for constructive interference.

A reinterpretation of the search in Sec. 2 can be done in terms of four-fermion CI in the particular case of the Helicity-Non-Conserving model, providing a limit on the preon binding energy scale $\Lambda$. The expected and observed 


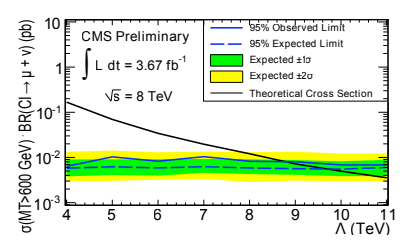

Figure 6. Bayesian limit for a $\mathrm{CI}$ in the muon channel.

limits for $\Lambda$ are shown in Figure 6. The lower limit on $\Lambda$ is $8.7 \mathrm{TeV}$.

\section{Search for excited leptons}

Compositeness models predict excited states of quarks and leptons at the characteristic scale of the new gauge interaction that binds preons. Since these excited fermions couple to SM fermions, they can be produced via CI and subsequently decay to SM fermions through the emission of a $\mathrm{W} / \mathrm{Z}$ boson or a photon. Previous searches have not found evidence for excited leptons. Here, the production of an excited lepton in association with an oppositely charged lepton of the same flavor, via four-fermion CI, is considered [11]. The excited lepton decay $\ell^{*} \rightarrow \ell \gamma$ results in the final state $\ell^{+} \ell^{-} \gamma$. The dominant, irreducible, SM BG in this search is the Drell-Yan production of $\ell^{+} \ell^{-} \gamma$ where the final state photon is either radiated by an initial-state parton (initial state radiation, ISR), or originates from one of the final-state leptons (final state radiation, FSR). The second-most important $\mathrm{BG}$ is due to $\mathrm{Z}+$ jets events where a jet is misidentified as a photon. Here, $\mathrm{Z}$ corresponds to the off-shell production as well. Another important BG is due to $\mathrm{W}+$ jets events where a jet is misidentitfied as an electron in the $\mathrm{e}^{*}$ channel. The analysis uses events with at least one well reconstructed primary vertex, one isolated high $p_{T}$ photon, and two isolated high $p_{T}$ leptons. The angle between the photon and the leading lepton, $\Delta R(\gamma, \ell)$, is required to be $\Delta R(\gamma, \mu)>0.7$ and $\Delta R(\gamma, \mathrm{e})>0.5$. The dilepton invariant mass is required to be above $60 \mathrm{GeV}$. For the muon channel, the dilepton invariant mass is required to be $25 \mathrm{GeV}$ above the nominal $\mathrm{Z}$ mass $(91.19 \mathrm{GeV})$. For the electron channel, the dielectron invariant mass and the electron-photon invariant masses are required to be outside $\mathrm{a} \pm 25 \mathrm{GeV}$ window centered at the nominal $\mathrm{Z}$ mass. The larger $\left(M_{\ell \gamma}^{\mathrm{Max}}\right)$ and the smaller $\left(M_{\ell \gamma}^{\mathrm{Min}}\right)$ invariant masses of the photon paired with one of the leptons are used for signal selection. A region in $M_{\ell \gamma}^{\mathrm{Min}}-M_{\ell \gamma}^{\mathrm{Max}}$ plane forming an L-shape is used for signal selection. For the chosen leptonphoton pairs, both invariant mass distributions peak at the excited lepton mass. The boundaries of the final selection depend on the excited lepton mass, and are identical for the excited electron and excited muon cases. For the lower masses, tighter L-shape selection regions are needed since the BG is more significant. For higher excited lepton masses the BG is negligible, a wider L-shape selection region is thus used. After all selection steps the expected BG is always below 2 events and decreases with the $\ell^{*}$ mass. The expected numbers of potential signal events and irreducible BG events are evaluated from simulation while the contribution of misidentified particles is derived from data. The uncertainties in the description of the detector performance, such as lepton energy or momentum resolution, lepton and photon energy scales, have been included in the systematic uncertainties. The impact on the signal yield corresponds to $\pm 2 \%$ and $\pm 3.5 \%$, respectively. A systematic uncertainty effect of $10 \%$ is conservatively assigned due to parton distribution functions on the BG. For masses above $0.4 \mathrm{TeV}$ no data events pass the selection criteria. Hence we provide upper limits on the production cross section of excited electrons and excited muons at the 95\% C.L. using a single bin counting method which takes into account the signal and BG yield uncertainties and the integrated luminosity normalization uncertainty. Limits for masses between 0.2 and $0.4 \mathrm{TeV}$ are higher due to the fact that the BG is located in this region. Assuming similar masses for $\mathrm{e}^{*}$ and $\mu^{*}$ the channels could be combined thus improving the excluded cross section limit to $0.73-$ $0.60 \mathrm{fb}$ for masses from $0.6 \mathrm{TeV}$ to $2 \mathrm{TeV}$. For each excited lepton mass, the excluded cross section can be associated to a value of the new interaction scale $\Lambda$. Excited electrons and muons with masses below $1.9 \mathrm{TeV}$ are excluded for the scale of CI $\Lambda=M_{\ell^{*}}$.
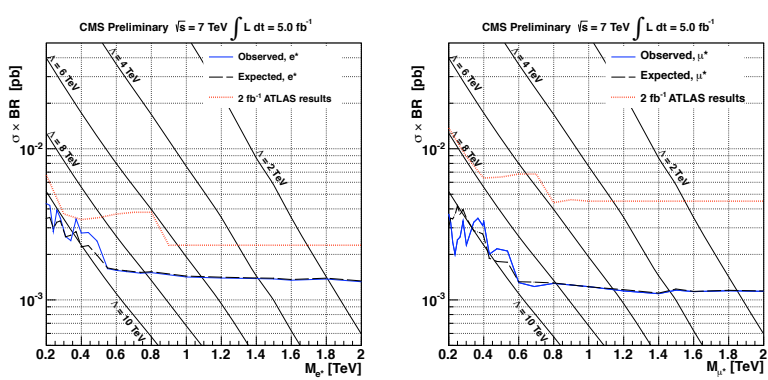

Figure 7. Expected and observed cross section upper limits (95\% C.L.) for different excited electron (left) and muon (right) mass points, using the $\mathrm{CL}_{\mathrm{s}}$ method. The black solid lines correspond to the excited lepton $\mathrm{LO}$ cross sections for different $\Lambda$ scales.

\section{References}

[1] C, Altarelli, G. and Mele, B. and Ruiz-Altaba, M., Z. Phys. 45, 109 (1989)

[2] Eichten, E. and Lane, K. and Peskin, M., Phys. Rev. Lett. 50, 811-814, (1983)

[3] Eichten, E. and Hinchliffe, I. and Lane, K. and Quigg, C., Rev. Modern Phys. 56, 579-707 (1984)

[4] CMS collaboration, CMS-PAS-EXO-12-010

[5] J. Phys. G, Nakamura, K and others, Particle Data Group 37, 075021 (2010)

[6] CMS collaboration, CMS-PAS-EXO-11-081

[7] Agashe et al., Phys.Rev. D76, 036006 (2007)

[8] CMS Collaboration, CMS-PAS-EXO-12-015

[9] CMS Collaboration, Phys.Lett. B716 82-102 (2012)

[10] CMS collaboration, CMS-PAS-EXO-11-009

[11] CMS Collaboration, CMS-PAS-EXO-11-034 